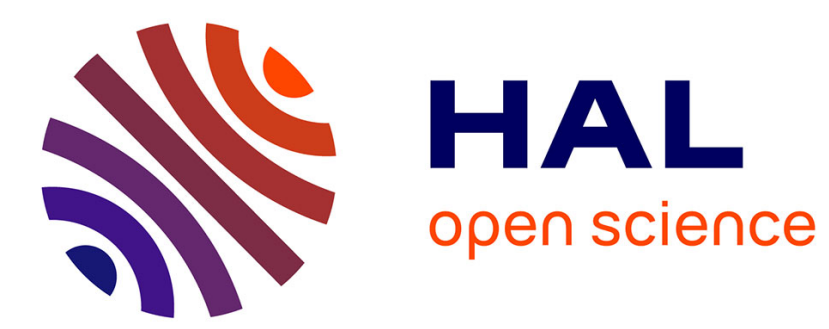

\title{
Read the labels
}

Christoph I Westbrook

\section{To cite this version:}

Christoph I Westbrook. Read the labels. Nature Physics, 2009, 5 (8), pp.538-539. 10.1038/nphys1348. hal-00487962

\section{HAL Id: hal-00487962 https://hal.science/hal-00487962}

Submitted on 31 May 2010

HAL is a multi-disciplinary open access archive for the deposit and dissemination of scientific research documents, whether they are published or not. The documents may come from teaching and research institutions in France or abroad, or from public or private research centers.
L'archive ouverte pluridisciplinaire HAL, est destinée au dépôt et à la diffusion de documents scientifiques de niveau recherche, publiés ou non, émanant des établissements d'enseignement et de recherche français ou étrangers, des laboratoires publics ou privés. 


\section{ATOM CHIPS}

\section{Read the labels}

Compact interferometers that exploit the wave character of atoms have the potential of outpacing their optical counterparts in a number of sensing applications. A technique that harnesses the internal structure of atoms should bring such applications a step closer.

\section{Chris Westbrook}

Atom interferometry holds great promise for the detection of inertial effects, acceleration, rotation and gravitation. Compared to optical interferometry, atoms have, for example, an intrinsic sensitivity to rotation, which is increased by the ratio of their rest energy to the energy of the photon - some ten orders of magnitude ${ }^{1}$. The last decade has seen the development of 'atom chips', devices in which atoms are trapped tens of micrometres above a solid substrate in the magnetic field created by wires on the surface ${ }^{2}$. A driving motivation for atom chips has been that compact, light and robust devices containing atom interferometers on a chip might, one day, fly to space or travel under the sea, bringing the power of atom interferometry to new environments. So one might dream of mapping out the gravitational field anywhere on Earth with centimetre resolution and part-per-billion accuracy in this way.

Although it has long been clear that an atom-chip interferometer would be important, building one has not been easy. On page 592 of this issue, Pascal Böhi and colleagues ${ }^{3}$ demonstrate an important innovation which should alleviate some of the practical problems that these devices face. They implemented on an atom chip a technique known as internalstate labelling, where different spatial paths in the interferometer are associated with different internal atomic states.

To be sensitive to inertial effects, it is necessary to divide atom wave functions into two spatially separated paths and to coherently recombine them. In addition, to read out the phase difference between the paths, one needs spatially resolved atom detection, another difficult task at the small scales of the chip. Internal-state labelling in the interferometer helps to avoid these problems. For one thing, it permits state-selective rather than positionselective detection. For alkali atoms, with their ground-state hyperfine structure, stateselective detection using resonant lasers - a technique that has been perfected in atomic 
clocks - is quite easy. Indeed, even in more macroscopic atom interferometers, internal state labelling has been a successful strategy ${ }^{1}$.

The experiment of Böhi et al. ${ }^{3}$ relies on two different hyperfine states, labeled 0 and 1 , which have nearly the same magnetic moment and which therefore experience nearly identical potentials in the magnetic fields created by the static currents on an atom chip (Fig. 1a). The main innovation in their experiments is the use of an appropriately tuned microwave field (at a frequency of $6.8 \mathrm{GHz}$, the hyperfine splitting of the rubidium-87 atom) which shifts the energy of the 0 state considerably more than that of the 1 state. In the near field that is, at distances much smaller than the wavelength - the microwave-field amplitude has a strong spatial gradient which translates into a strong gradient in the energy of the 0 state. This gradient displaces the equilibrium position of state 0 by about $5 \mu \mathrm{m}$ relative to the 1 state (Fig. 1b). The microwave potential is switched on suddenly and thus the 0 state begins to oscillate in its new potential, returning after every $8.6 \mathrm{~ms}$ period to its initial position, where it is superimposed with the 1 state.

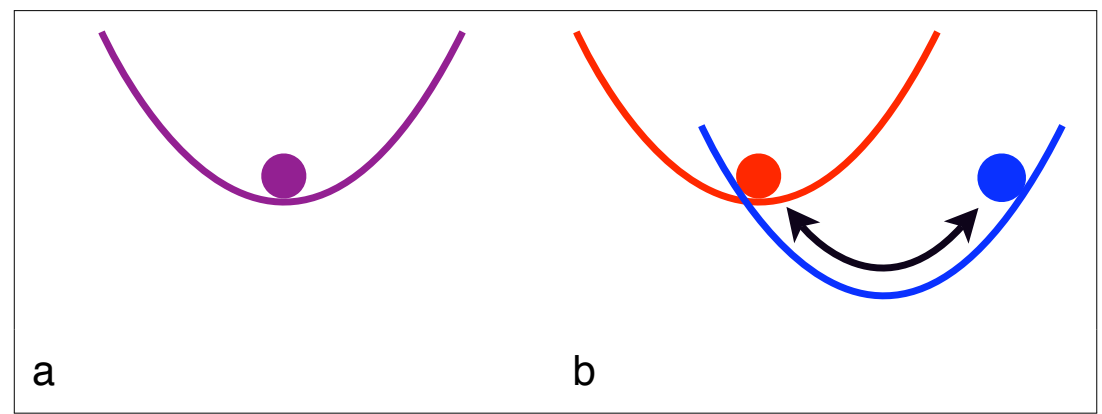

Figure 1 I An interferometer with internal-state labelling. An atom can be in two internal quantum states, 0 (blue) and 1(red). a, Initially the two states feel the same potential (purple line), and a quantum superposition of internal states corresponds to a spatial overlap of the wave functions of the atoms (purple dot). $\mathbf{b}$, If the potential felt by the 0 state (blue line) is suddenly changed, the 'blue part' of the wave function begins to oscillate, and returns to overlap with the red part after one period. When the atoms overlap, the 0 and 1 states can be temporarily coupled and the final population in 0 and 1 varies according to the phase difference acquired while the states were separated. In the experiment of Böhi et al. ${ }^{3}$, the coupling and the change in potential are produced by microwave fields. Although they are spatially overlapped at the output, the two states are easily distinguished by resonant lasers. 
Before the application of this microwave separation pulse, the atoms are put into a superposition of the 0 and 1 states, and after the separation pulse, the two states are recombined. As with any interferometer, the final population in the possible output states depends on the acquired phase difference between the different interferometer paths. Böhi et $a l .{ }^{3}$ observe interference fringes with a good signal-to-noise ratio after a separation corresponding to an oscillation in the 0 state potential. This phase difference can depend on the accelerations undergone by the atoms during their separation. One could also imagine introducing and measuring other phase shifts in such an interferometer, due to atom-surface interactions, for instance.

But the experiment can also be viewed, in the context of quantum information processing, as one half of a two-bit quantum gate. If two such interferometers were placed side by side on a chip, the paths could be arranged in such a way that the atoms collide if they are both in the 0 state. If the atoms in each interferometer are in a superposition of the 0 and 1 states, the resulting conditional, elastic collision results in a conditional phase shift which entangles the internal states of the two atoms ${ }^{4}$.

We are not quite ready to put devices like this one into submarines. The 'intrinsic' advantage of atoms over photons in interferometric inertial sensing mentioned above neglects the fact that parameters such as the enclosed area and the particle flux also play a large role in determining the sensitivity of an interferometer. To realize its potential, the spatial separation and the number of atoms involved (in the experiment of Böhi et al. ${ }^{3}$ there were 3000 of them) needs to be vastly increased. A second important problem for atom-chip interferometry is uncontrolled dephasing between the interferometer paths. The proximity to the surface, which the authors use to such advantage here, also enhances the sensitivity of the atoms to thermal currents (so-called Johnson noise) on the chip ${ }^{5}$. Careful work is still needed to learn how these difficulties can be overcome. For purposes of quantum information processing, finally, the sensitivity to environmental noise is also of capital importance, although this application will impose different constraints. But given the ingenuity shown by the community so far, I would be surprised if there wouldn't be many more advances in store for us.

Chris Westbrook is in the Laboratoire Charles Fabry de l'Institut d'Optique, Campus Polytechnique, 91127 Palaiseau, France.

e-mail: christoph.westbrook@institutoptique.fr 


\section{References}

1. Cronin, A. D., Schmiedmayer, J. \& Pritchard, D. E. Rev. Mod. Phys. (in the press).

2. Fortágh, J. \& Zimmermann, C. Rev. Mod. Phys. 79, 235-289 (2007).

3. Böhi, P. Nature Phys. 5, 592-597 (2009).

4. Carlarco, T. et al. Phys. Rev. A 61, 022304 (2000).

5. Fermani, R., Scheel, S. \& Knight, P. L. Phys. Rev. A. 73, 032902 (2006). 